\title{
Chiasma distribution in the first bivalent of mice carrying a double insertion of homogeneously-staining regions in homo- and heterozygous states
}

\author{
IVAN P. GORLOV*, TATYANA YU. LADYGINA \& PAVEL M. BORODIN \\ Institute of Cytology and Genetics, Academy of Sciences of the U.S.S.R., Siberian Department and Department of \\ Cytology and Genetics of the Novosibirsk State University, Novosibirsk 630090, U.S.S.R.
}

\begin{abstract}
An examination of the meiotic pattern of chromosome 1 isolated from a feral mouse population and containing a double insertion (Is) of homogeneously-staining regions (HSRs) was carried out. In a previous study is was shown that the region delineated by the proximal breakpoint of Is(HSR;1C5) $1 \mathrm{Icg}$ and the distal one of Is(HSR;1D)2Icg is unpaired during early pachytene and heterosynapsed at midpachytene. No synaptic disturbances were revealed in homozygotes in this study. Chiasmata number per first bivalent in heterozygous (1.87) and homozygous (1.88) males was shown to be higher than in normal ones (1.61). In normal males a single chiasma is located in the medial part of chromosome 1 . In heterozygotes this segment is heterosynapsed and unavailable for recombination. This leads to a significant decrease in the frequency of bivalents bearing a single chiasma and an increase in the frequency of bivalents bearing double chiasmata located mostly at subcentromeric and subtelomeric regions of the chromosome. In homozygous males the frequency of double chiasmata is also increased, and even triple chiasmata become possible because of the increase in the physical length of the bivalents. Thus insertion of heterochromatic regions, which are inert with respect to recombination, leads to an increase in the length of the genetic map of the chromosome because of relaxation of interference restrictions.
\end{abstract}

Keywords: chiasma, insertion, interference, meiosis, mouse, synapsis.

\section{Introduction}

The mechanisms controlling chiasma frequency and distribution are not clear. Different levels of determination of these characters could be supposed: organismal, cellular and chromosomal. At the chromosomal level the leading role is played by such factors as physical size of the chromosome and efficiency of pairing in certain regions of it. It has been shown that it is possible to model the frequency and position of chiasmata for each bivalent in terms of its length and interference distance (Mather, 1937, 1938; Henderson, 1963; Fox, 1973; Gorlov, et al., 1987). Besides interference and physical length of the chromosome there are some mechanisms of precise control of chiasma position in certain chromosomal regions. For example, suppressive effects of synaptic disturbances on recombination

*Correspondence. are well known (Holliday, 1977). Lack of crossing over in heterochromatic regions has been shown in different species (Stack, 1984). Pairing in heterochromatic, later replicating regions might be ineffective in providing the stringent requirement of the crossover process (John, 1976; Chandley, 1986).

It is difficult to separate the relative impact of these factors in controlling chiasma frequency and distribution on the basis of interspecies or interchromosomal comparisons.

Chromosome 1 bearing double insertion of homogeneously staining regions (HSR) isolated from a feral population of the house mouse (Mus musculus musculus) (Agulnik et al., 1987, 1988) is a convenient model for the investigation of these problems. These insertions Is(HSR;1C5)1Icg and Is(HSR;1D)2Icg are C-positive, their total length is about 50 per cent of normal chromosome 1 size. They are located in the middle part of the chromosome and separated by a 
small inverted euchromatic region (Agulnik et al., 1987, 1988). A single insertion of HSR variable with respect to its size, and localized at the band $1 \mathrm{C} 5$, has been isolated from European populations of $M . \mathrm{m}$. domesticus (Traut et al., 1984). The HSR contains amplified and probably rearranged DNA sequences (Boldyreff et al., 1988). These data allow us to suppose that the double insertion of HSR is a result of inversion arising in chromosome 1 carrying a single insertion of HSR. The breakpoints of the inversion are located inside the HSR and in the E3 subband (Agulnik et al., 1990).

Using this chromosome it is possible to estimate the effect of the local non-homology on chromosome pairing and on chiasma frequency and distribution. In the previous study (Borodin et al., in 1990) it was shown that the region delineated by the proximal breakpoint of Is(HSR;1C5) $1 \mathrm{Icg}$ and the distal one of Is(HSR;1D)2Icg was unpaired during early pachytene and unavailable for recombination. This leads to a significant decrease in the frequency of bivalents bearing single chiasmata and an increase in the frequency of bivalents bearing double chiasmata, located mostly at subcentromeric and subtelomeric regions of the chromosome.

The aim of this study was an electron microscopic (EM) study of chromosome pairing and light microscopic (LM) analysis of chiasma frequency and distribution in male mice homozygous for a double insertion of HSRs in comparison with heterozygous and normal ones. This model allows one to estimate the roles of the physical length of the chromosome and an additional heterochromatic region in the control of chiasma frequency and distribution.

\section{Materials and methods}

Chromosome 1 containing insertions Is(HSR; 1C5)1Icg and Is(HSR;1D)2Icg is maintained on the background of the CBA/Lac strain possessing chromosome 1 marked by a small block of subcentromeric C-heterochromatin (Forejt, 1973).

EM analysis of surface-spread pachytene nuclei and LM study of diplotene-diakinetic cell were performed on three homozygous, three heterozygous and three normal adult males which were obtained from the crosses of heterozygous animals.

Somatic karyotypes (Fig. 1A) were characterized on metaphases prepared from bone marrow biopsy. Testicular material for meiotic preparations was obtained from adult males. Diplotene-diakinesis chromosome spreads (Fig. 1B) were prepared after hypotonic treatment and methanol:acetic acid (3:1) fixation by a routine air-drying technique (Evans et al., 1964). For identification of HSR and subcentromeric C-heterochromatin in chromosome 1 , slides were processed by C-band staining technique (Sumner, 1972).

Well-spread diplotene and diakinetic nuclei were traced using a drawing apparatus. Each bivalent of chromosome 1 marked by either an HSR or a small precentromeric $\mathrm{C}$-band was subdivided into five parts according to its relative size, as shown in Table 1, and chiasmata locations were established.

Spread preparations of synaptonemal complexes (SC) (Fig. 1C) for EM analysis from spermatocytes were made following the technique described by Moses (1980), stained with $\mathrm{AgNO}_{3}$ (Howell \& Black, 1980) and examined by an EMF(U.S.S.R.) electron microscope at $75 \mathrm{kV}$. Meiotic prophase nuclei were sub-
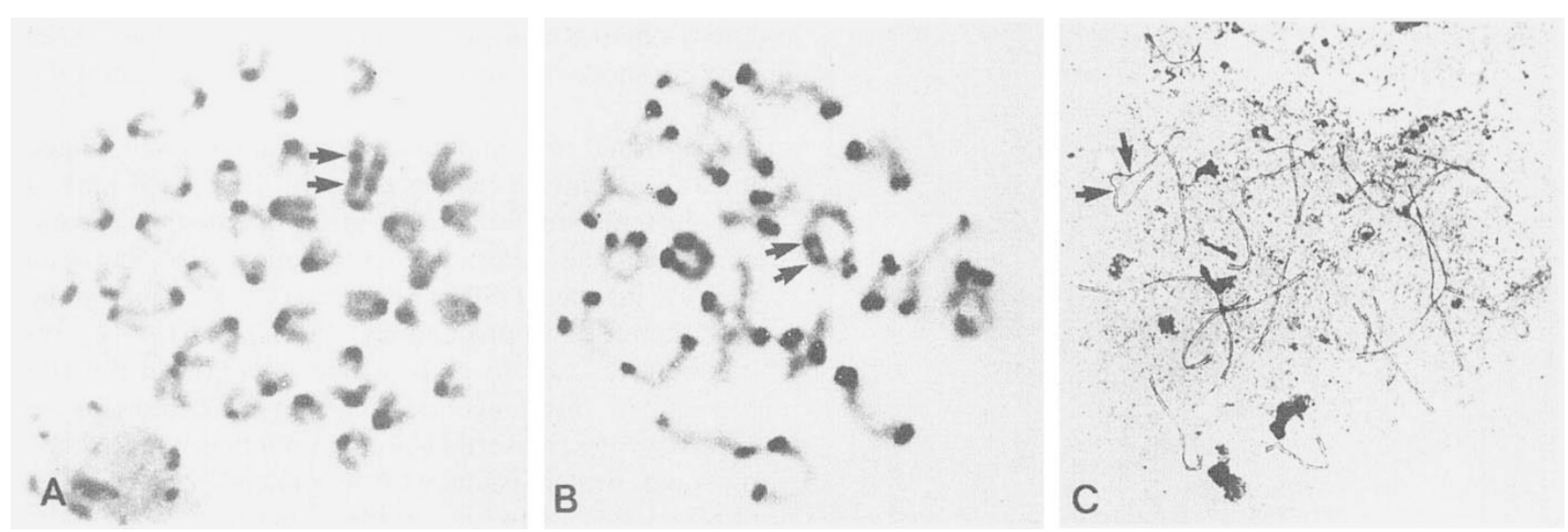

Fig. 1 Karyotypes of male mice heterozygous for insertions of the homogeneously-staining C-positive regions Is(HSR;1C5)1Icg and Is 9HSR;1D)2Icg; arrows show the breakpoints of rearrangements in chromosome 1. (A) Metaphase plate (C-band staining). (B) Diakinetic chromosomes (C-band staining). (C) Synaptic configuration from a pachytene cell of male mouse heterozygous for chromosome 1 with double heterochromatic insertions. 


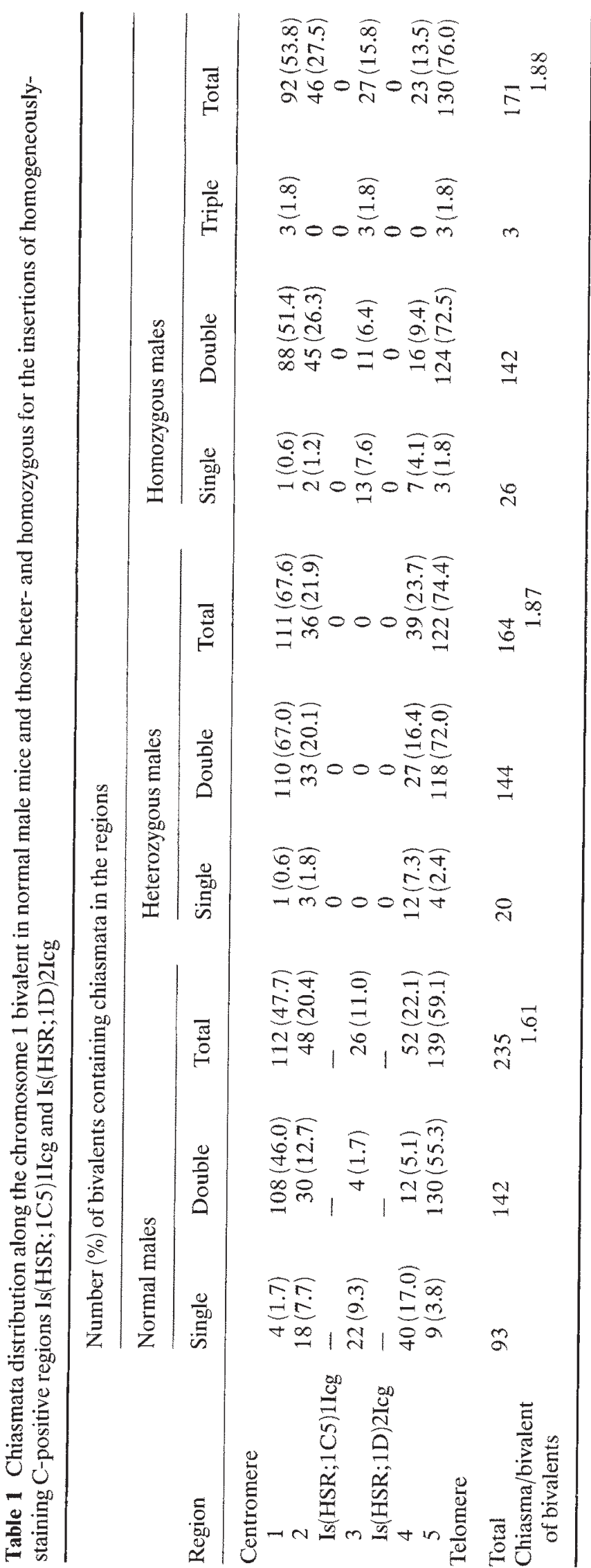

staged according to criteria established by Moses (1980).

\section{Results}

\section{Synapsis}

A total of 200 silver-stained nuclei of primary spermatocytes from three homozygous males and 200 from three normal males were scored at EM level for the presence or absence of possible insertion/deletion loop configurations in the chromosome 1 bivalent. Such loops were not observed in normal and homozygous animal nuclei.

It has been shown earlier (Borodin et al., 1990) that only 4.4 per cent of spermatocytes from heterozygous males contain a single unpaired D-loop in the chromosome 1 bivalent. All these nuclei were at the stage of late zygotene-early pachytene. Those without loops were at later stages of pachytene. The disappearance of loops between early pachytene and later pachytene substages is presumably the result of synaptic adjustment, which has been frequently reported in the mouse (Moses, 1980). Accommodation involves nonhomologous pairing (heterosynapsis) of this region of chromosome 1. No inversion loops have been observed in heterozygotes. The lack of inversion loops is probably determined by the short length of inverted region and/or by the synaptic failure in adjacent HSRs.

\section{Chiasma frequency and location}

$\mathrm{C}$-band diplotene and diakinetic nuclei were scored from 235 normal, 164 heterozygous and 171 homozygous males. The sample size of cells from normal and heterozygous males was increased in comparison with previously published data (Borodin et al., 1990).

No obvious chiasmata were revealed in the HSRs. Table 1 shows the distributions of chiasmata along the bivalent.

In normal and in homozygous males single chiasmata are located predominantly in the medial part of the chromosome. In heterozygotes the medial segment containing the inverted euchromatic region between the two inserted HSRs is unpaired at early pachytene and therefore is presumably unavailable for recombination. It leads to a significant decrease in the frequency of bivalents bearing single chiasmata in heterozygotes. Nevertheless, the total number of chiasmata per bivalent is much higher in heterozygous males $(1.87 \pm 0.03)$ than in normal males $(1.61 \pm 0.03)$ $(P<0.05)$. The increase in the total chiasmata number in the heteromorphic bivalent results from the addition of double chiasmata located mostly at subcentromeric and subtelomeric regions of the chromosome. 
Homozygotes do not differ from heterozygotes in the total chiasmata number $(1.88 \pm 0.03)$, but have significantly higher numbers than normal males $(1.61 \pm 0.03)(P<0.05)$.

No synaptic disturbances have been revealed in the medial part of the bivalent in homozygous males. The total number of chiasmata in this region is approximately the same as in the normal males, although no chiasmata were observed in the HSRs.

The ratio of single to multiple chiasmata in the medial region changes in favour of multiple ones. For example, the formation of triple chiasmata takes place only in homozygotes, and all of them are located in subcentromeric, medial and subtelomeric regions.

\section{Discussion}

In earlier genetic studies, so called K-factors (Sturtevant, 1926) have been revealed. They suppress recombination in the heterozygous state and have no effect in homozygotes. This is a property of small inversions (Novitski \& Braver, 1954), translocations (Roberts, 1970), duplications (Kalisch, 1975) and deletions and transpositions (Rhoades, 1968). The results of our experiment demonstrate the same effect of inverted duplication of the additional heterochromatic segment. It suppresses recombination in affected regions in heterozygotes and has no effect in homozygotes.

Polymorphism for additional heterochromatic segments has been found in some species of grasshoppers (John \& King, 1985). These heterochromatic blocks restrict the chiasmata in adjacent euchromatic segments. The presence of terminally-located heterochromatic segments leads to a substantial redistribution of chiasmata away from the segments to more proximal sites. In mice the insertions of additional heterochromatin do not suppress recombination in neighbouring regions. Even in the interstitial euchromatic region delineated by the two blocks of additional heterochromatin we do not observe any decrease of chiasma frequency. The suppressive effect of the insertion is not revealed either in the proximal or distal regions flanking the insertions. The main finding of this study is the increase in the length of the genetic map of chromosome 1 after insertion of recombinationallyinert chromatin. This increase has been observed both in homo- and in heterozygotes. The genetic length of normal chromosome 1 estimated from chiasma frequency is $80 \mathrm{cM}$. In homo- and heterozygotes for chromosome 1 , bearing the insertions Is(HSR; 1C5) $1 \mathrm{Icg}$ and Is(HSR;1D)2Icg, this increase to approximately $94 \mathrm{cM}$. The gain is about 18 per cent of the genetic length of normal chromosome. Does it correspond to the increase of physical size of the chromosome as a result of the insertions?
The insertion increases the total length of chromosome 1 in mitotic metaphase by 50 per cent (Agulnik et al., 1987, 1988). However, because of unproportional elongation of eu- and heterochromatic regions the length of pachytene in chromosome 1 carrying the HSRs either in homozygous or in heterozygous states increases not by 50 per cent but by 25 per cent (Gorlov, unpublished data). The degree of increase of the physical and the genetic length of the chromosome is rather similar.

However, we must take into account that the chromatin of the inserted regions is inert with respect to recombination: no chiasmata have been observed in these regions. This latter finding is not surprising, because of the heterochromatic nature of the inserted regions (Agulnik et al., 1987, 1988). It has been suggested that the heterochromatic late-replicating regions are ineffectively synapsed and not available for recombination (Stack, 1984; Chandley, 1986).

The genetic length of the chromosome also increases in heterozygotes, despite the fact that in this case the interstitial region of euchromatin, amounting to 10 per cent of pachytene chromosome 1 length and $7 \mathrm{cM}$ genetic map length in homozygous and normal males, is excluded from recombination.

The addition of heterochromatic regions to the chromosome does not increase the number of additional sites of crossing over. How, in this case, can they lead to the increase in recombinational length of the chromosome? The question of special interest is: why is the degree of increase the same in the heteroand homozygous states? It is suggested that the insertions relax the interferential restriction to the occurrence of 'secondary' chiasmata. However, it is hard to discuss the causes of this relaxation when we have no reliable idea of the mechanism of chiasma interference. Several hypotheses for explaining this phenomenon have been advanced. It has been supposed that the formation of chiasmata is determined by a special factor: 'recombinase' (King, 1970) or 'chiasma determination mechanism' (Fox, 1973) moving along the chromosome. Some time after, and consequently at some distance from, the first chiasma the factor is unable to form a second one. From this point of view, interference can be determined as the distance of shift of the 'exhausted' enzymatic complex. Other authors suppose that interference is realized through the factor suppressing new recombination events close to the first arisen chiasma (Wettstein et al., 1984) or it is a result of competition between potential sites of recombination for DNA-binding protein, diffusing freely within the SC (Holliday, 1977). The protein supply is exhausted at some distance from the first recombination nodule and no second chiasma can be formed. According to Holliday, the lack of interference 
across the centromere results from the SC structure at the centromere which does not allow the protein to diffuse through it. Altered SC structure has been described not only in the centromeric heterochromatic regions but also in intercaller heterochromatin (Stack, 1984).

SC obviously plays an important role in interference (Egel, 1978). Olson et al. (1978) and Egel-Mitani et al. (1982) have not found any SC in yeasts which are characterized by the absence of interference. Introduction of SC discontinuities and the consequent creation of independent pairing segments are the causes of structural rearrangements: translocations (Arana et al, 1986; Sybenga, 1970), centric fissions and interchanges (Parker et al., 1982; Parker, 1987) lead to abolition of interference.

The above mentioned hypotheses present the factor of interference as a diffusible entity that can be characterized by the diffusion distance and by the conductibility of a channel. From this point of view, we can try to explain our findings.

In insertion homozygotes, the additional length or probable altered structure of SC of heterochromatic segments may prevent or slow down the transmission of the interference factor and thus reduce interferential interaction between the potential sites of crossing over. In heterozygotes, on the other hand, asynapsis of the medial region carrying two insertions and inversion results in an SC discontinuity, which may be the critical factor in preventing the transmission of the interference factor between proximal and distal segments of the bivalent.

The conclusion from this discussion is not very optimistic. We have not reached an understanding of the nature of interference, as was the case 80 years ago. However, we have revealed an interesting effect of this mysterious phenomenon on the size of genetic maps: they can be increased by insertions of recombinationally-inert chromatin.

\section{Acknowledgements}

The research was supported by the Russian State Programme 'Frontiers in Genetics'. We are extremely grateful to an anonymous reviewer for helpful comments on the manuscript and to Mrs Marina I. Rodionova for technical assistance.

\section{References}

AGULNIK, S. I., BORODIN, P. M., GORLOV, I. P., LADYGINA, T. YU. AND PAK, S. D. 1993. Causes of appearance of a double insertion of homogeneously staining regions in the house mouse (Mus musculus musculus). Heredity, 65, 265-271.
AGULNIK, S. 1., GORLOV, 1. P. AND AGULNIK, A. I. 1987. Mouse Chr I with two insertions: Is(HSR; 1C5)1Icg and Is(HSR; 1E3)2Icg. Mouse News Lett., 78, 70.

AGULNIK, S. I., GORLOV, I. P. AND AGULNIK, A. I. 1988. New variant of chromosome 1 of the house mouse. Cytologiya, 30, 773-775 (in Russian).

ARANA, P., SANTOS, J. L. AND GIRALDEZ, R. 1980. Chiasma interference and centromere co-orientation on spontaneous translocation heterozygote of Eucherthippus pulvinatus gallicus (Acrididae; Orthoptera). Chromosoma, 78, $327-340$.

BOLDYREFF, B., WEITH, A., WINKING, H., PARMANN, L. AND TRAUT, W. 1988. Amplification and rearrangement in a germ line HSR of the mouse. Genet. Res., 52, 65-70.

BORODIN, P. M., GORLOV, I. P. AND LADYGINA, T. YU. 1990. Double insertion of homogeneously staining regions in chromosome 1 of wild Mus musculus musculus: effects on chromosome pairing and recombination. J. Hered., 81, 91-95.

CHANDLEY, A. C. 1986. A model for effective pairing and recombination at meiosis based on early replicating sites (R-bands) along chromosomes. Hum. Genet., 72, 50-57.

EGEL, R. 1978. Synaptonemal complex and crossing over: structural support or interference? Heredity, 43, 233-237.

EGEL-MITAN1, M., OLSON, L. W. AND EGEL, R. 1982. Meiosis in Aspergillus nidulans: another example for lacking synaptonemal complexes in the absence of crossover interference. Hereditas, 97, 179-188.

EVANS, E. P., BRECKON, G. AND FORD, C. D. 1964. An air-drying method for meiotic preparations from mammalian testes. Cytogenetics, 3, 289-299.

FOREIT, J. 1973. Centromeric heterochromatin polymorphism in the house mouse. Evidance from inbred strains and natural populations. Chromosoma, 43, 187-201.

Fox, D. P. 1973. The control of chiasma distribution in the locust, Schistocerca gregaria (Forskal). Chromosoma, 65, 247-267.

GORLOV, I. P., AGULNIK, A. I. AND AGULNIK, S. I. 1987. Localization of house mouse genes on chromosomes via comparison of the genetic map and the chiasmata distribution. Genetika, 23, 63-70 (in Russian).

HENDERSON, S. A. 1963. Chiasma distribution at diplotene in a locust. Heredity, 18, 173-190.

HOLLIDAY, R. 1977. Genetic recombination and meiosis. Philos. Trans. R. Soc. London B., 277, 359-370.

HOWELL, W. M. AND BLACK, D. A. 1980 . Controlled silver-staining of nucleolus organizer-regions with protective colloidal developer: a 1-step method. Experientia, 36, 1014-1015.

JонN, B. 1976. Myth and mechanisms of meiosis. Chromosoma, 54, 295-325.

JOHN, W. AND KING, M. 1985. The inter-relationship between heterochromatin distribution and chiasma distribution. Genetica, 66, 183-194.

KALISCH, w. E. 1975. Tandem duplication in Drosophila melanogaster. II. Meiotic pairing and exchange in heterozygous tandem duplications. Theoret. Appl. Genet., 46, $169-180$.

KING, R. C. 1970 . The meiotic behaviour of the Drosophila oocyte. Int. Rev. Cytol., 28, 125-165. 
MATHER, K. 1937. The determination of position in crossingover. II. The chromosome length-chiasma frequency relation. Cytologia Fujii Jubilee Vol., 514-526.

MATHER, K. 1938. Crossing-over. Biol. Rev., 13, 252-292.

MOSES, M. J. 1980. New cytogenetic studies on mammalian meiosis. In: Serio, M. and Martini, L. (eds) Animal Models in Human Reproduction. Academic Press, London, pp. 169-190.

NOVITSKI, E., AND BRAVER, G. 1954. An analysis of crossing over within a heterozygous inversion in Drosophila melanogaster. Genetics, 39, 197-209.

OLSON, L. W., EDEN, U., EGEL-MITANI, M. AND EGEL, R. 1978. Asynaptic meiosis in fission yeast? Hereditas, 89, 189-199.

PARKER, J. S. 1987. Increased chiasma frequency as a result of chromosome rearrangement. Heredity, 58, 87-94.

PARKER, J. S., PALMER, R. W., WHITEHORN, M. A. F. AND EDGAR, L. A. 1982. Chiasma frequency effects of structural chromosome change. Chromosome, 85, 673-686.

RHOADES, M. M. 1968. Studies on the cytological basis of crossing-over. Peacok, W. J. and Brock, R. D. (eds). Replication and Recombination of Genetic Material. Australian Academy of Science, Canberra, pp. 229-241.
ROBERTS, P. A. 1970. Screening for X-ray-induced crossover suppressors in Drosophila melanogaster: prevalence and effectiveness of translocations. Genetics, 65, 429-448.

STACK, S. 1984. Heterochromatin, the synaptonemal complex and crossing-over. J. Cell Sci., 71, 159-176.

Sturtevant, A. H. 1926. A crossover reducer in Drosophila melanogaster due to an inversion of a section of the third chromosome. Biol. Zenir. Bl., 46, 697-702.

SUMNER, A. T. 1972. A simple technique for demonstrating centromeric heterochromatin. Exp. Cell Res., 75, 304-306.

SYBEnGA, J. 1975. Meiotic configurations. In: Frankel, R. (ed.). Monographs on Theoretical and Applied Genetics 1. Springer Verlag, Berlin, pp. 1-251.

TRAUT, W., WINKING, H. AND ADOLPH, s. 1984. An extra segment in chromosome 1 of wild Mus musculus: C-band positive homogeneously staining region. Cytogenet. Cell Genet., 38, 290-297.

WETTSTEIN, P., RASMUSSEN, S. W. AND HOLM, P. B. 1984. The synaptonemal complex in genetic segregation. Ann. Rev. Genet., 18, 331-413. 